\title{
Algoritmo para determinação do amortecimento de materiais cerâmicos pela técnica das freqüências naturais de vibração via excitação por impulso
}

\section{(Algorithm to determine the damping of ceramic materials by the impulse excitation technique)}

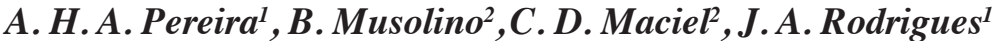 \\ ${ }^{I}$ Grupo de Engenharia de Microestrutura de Materiais - GEMM, Departamento de Engenharia de Materiais, \\ Universidade Federal de S. Carlos, Rod. Washington Luiz, km 235, S. Carlos, SP 13565-905 \\ ${ }^{2}$ Departamento de Engenharia Elétrica, Escola de Engenharia de S. Carlos, Universidade de S. Paulo, \\ S. Carlos, SP 13566-590 \\ ha@atcp.com.br, josear@ufscar.br
}

\begin{abstract}
Resumo
O amortecimento ou atrito interno é uma das propriedades mais sensíveis de materiais e estruturas tanto em escala macro como microscópica e um bom indicador para a caracterização de danos estruturais, sendo particularmente sensível à presença de trincas e micro-trincas. O objetivo deste trabalho foi desenvolver um algoritmo que permitisse incorporar a caracterização do amortecimento à técnica das freqüências naturais de vibração via excitação por impulso, já bem estabelecida pela norma ASTM E1876 e amplamente empregada na determinação não-destrutiva dos módulos elásticos de materiais cerâmicos. Foi desenvolvido um algoritmo para a determinação do amortecimento fundamentado na análise de sinal no domínio de tempo-freqüência, no método do decremento logarítmico e no modelo viscoelástico equivalente. O algoritmo foi validado empregando um gerador de ondas de formato arbitrário e aplicado na caracterização de dois materiais, sendo um concreto refratário de alta alumina, com e sem dano por choque térmico, e um aço SAE 1020. O algoritmo foi eficiente ao determinar a frequência e o amortecimento de sinais arbitrários e dos corpos de prova, e em limitar a análise ao modo de vibração de interesse, minimizando assim a interferência de outros modos. O algoritmo permitiu integrar com eficiência o cálculo do amortecimento à técnica de excitação por impulso.

Palavras chave: algoritmo, amortecimento, materiais cerâmicos, freqüências naturais, ASTM E1876.
\end{abstract}

\begin{abstract}
Damping is one of the most sensitive properties of materials and structures, in macro and microscopic scale, which makes it particularly sensitive to the presence of cracks and micro-cracks and a good parameter to the characterization of structural damages. The aim of this work was to develop an algorithm to allow the incorporation of the damping characterization to the natural frequencies of vibration technique (by impulse excitation), already well established for the non-destructive determination of elastic moduli by ASTM E1876 and correlated standards for ceramic materials. It was developed an algorithm for determining the damping based on the time-frequency domain signal analysis, logarithmic decrement method and on the equivalent viscoelastic model. The algorithm was validated using an arbitrary wave form signal generator and applied to characterize two materials, a high alumina refractory castable with and without thermal shock damage, and a SAE-1020 steel. The algorithm was effective to recovery the frequencies and respective damping of the arbitrary simulated signals and to characterize the specimens, limiting the analysis to the vibration mode of interest, thereby minimizing the modes interference. This algorithm allowed the successful integration of the damping characterization to the impulse excitation technique.
\end{abstract}

Keywords: algorithm, damping, ceramic materials, natural frequencies, ASTM E1876.

\section{INTRODUÇÃO}

O amortecimento ou atrito interno é uma das propriedades mais sensíveis de materiais e estruturas, tanto em escala macro quanto microscópica [1], sendo particularmente sensível à presença de trincas e micro-trincas [2]. Além da aplicação clássica no estudo de metais e nas áreas de engenharia mecânica e engenharia civil, a caracterização do amortecimento também vem sendo empregada no estudo de materiais cerâmicos refratários para a avaliação do dano por choque térmico $[3,4]$. A tensão mecânica induzida pelo gradiente de temperatura do choque térmico provoca a nucleação e propagação de micro-trincas e trincas que degradam as propriedades mecânicas do material determinando em grande parte a sua vida útil $[5,6]$. A nucleação e evolução destas micro-trincas e trincas podem ser monitoradas com a caracterização do amortecimento [3, $4,7]$. A caracterização do amortecimento também pode ser 
empregada para o controle de qualidade em linhas de produção. O amortecimento é o fenômeno pelo qual a energia mecânica de um sistema dinâmico é dissipada [8]. O amortecimento de um sistema ou material pode ser classificado de três formas principais: interno, estrutural e fluídico. O interno está associado aos defeitos na microestrutura, efeitos anelásticos e a efeitos termoelásticos causados por gradientes locais de temperatura. Já o estrutural está associado a perdas de energia por atrito em juntas, parafusos e articulações semi-rígidas. Por último, o fluídico ocorre por resistência ao arraste em meio fluídico, por exemplo, a conversão de energia cinética de um pêndulo em energia térmica para o ar. Neste trabalho o interesse é pela avaliação do amortecimento interno ou atrito interno [8].

Existem diversos métodos para a determinação do amortecimento, sendo os mais utilizados em análise modal os do decremento logarítmico e da largura de banda de meia potência $[9,10]$. A escolha do método depende da faixa do amortecimento, da frequência de vibração, da praticidade requerida e do hardware disponível $[8,11]$. O método do decremento logarítmico é mais adequado para baixo e médio amortecimento e consiste no cálculo a partir da atenuação da vibração do material ou estrutura em função do tempo após uma excitação por impulso. $\mathrm{O}$ método da largura de banda de meia potência é mais adequado para médio e alto amortecimento. $\mathrm{O}$ método do decremento logarítmico é o mais prático, contudo demanda de uma capacidade superior de processamento de sinal. Ambos os métodos consideram um modelo para os cálculos, normalmente o modelo de amortecimento viscoelástico equivalente, em que o decremento da amplitude de vibração em função do tempo é tido como exponencial e o amortecimento não depende da freqüência de vibração $[8,11,12]$. A técnica das freqüências naturais de vibração via excitação por impulso consiste essencialmente em excitar, através de um leve e breve impacto mecânico, o modo de ressonância flexional e/ou torcional fundamental de uma amostra em formato de barra, e em calcular os módulos elásticos dinâmicos [13] empregando as equações de Pickett [14] a partir das frequências encontradas na resposta acústica, das dimensões e da massa da amostra. A pancada deve ser curta o suficiente para que apresente um espectro plano de frequência na faixa de interesse. O corpo de prova reage à pancada como um filtro passa-faixas revelando as suas freqüências naturais de vibração através da emissão de uma resposta acústica transitória, que também contém a informação para a determinação do amortecimento pelo método do decremento logarítmico. A excitação do modo de vibração desejado é realizada impondo-se condições de contorno mecânicas e de excitação adequadas, o que consiste em apoiar o corpo de prova nas linhas nodais de vibração e excitá-lo onde a amplitude de vibração será máxima [15]. Contudo, dificilmente se consegue excitar um único modo de vibração, mesmo porque quando é desejado se obter a razão de Poisson é necessário excitar modos flexionais e torcionais. Em função desta dificuldade, a aplicação do método do decremento logarítmico padrão (no domínio do tempo) possui um componente de incerteza significativa associada à contribuição para a resposta acústica de modos de vibração menos favorecidos pelas condições de contorno, visto que estes modos apresentam maior interação com o suporte de corpo de prova. Estes modos menos favorecidos também aumentam a incerteza dos módulos elásticos quando o cálculo da frequência realizado pelo sistema de medição é feito através do período do sinal captado, como acontece em métodos fundamentados em zero-crossing [16].

O objetivo deste trabalho foi desenvolver um algoritmo integrável à técnica de excitação por impulso que contornasse os problemas mencionados. O desenvolvimento do algoritmo foi fundamentado na análise de sinal no domínio tempo-freqüência, através da qual o decremento logarítmico da amplitude é obtido e avaliado exclusivamente para a frequência correspondente ao modo de vibração mais favorecido pelas condições de contorno, garantindo, assim, o cálculo do amortecimento com maior precisão. Este recurso é particularmente bem vindo no caso de materiais cerâmicos com alto dano microestrutural, por exemplo, concretos refratários submetidos a choques térmicos e concretos estruturais submetidos a cargas elevadas, nos quais o dano aumenta o amortecimento de forma expressiva o que degrada a razão sinal/ruído e aumenta o acoplamento entre os modos de vibração. A conveniência de unir o método do decremento logarítmico no domínio tempo-freqüência à técnica das frequências naturais via excitação por impulso reside no fato da resposta acústica conter a informação necessária para a determinação do amortecimento, e no fato da geometria do corpo de prova e as condições de contorno do ensaio serem padronizadas [13]. Adicionalmente, a incorporação da medição do amortecimento não demanda de procedimentos experimentais adicionais ou altera a duração do ensaio, mantendo assim a praticidade e rapidez da técnica.

\section{MATERIAIS E MÉTODOS}

\section{Modelo viscoelástico equivalente}

O modelo viscoelástico equivalente [8] é o alicerce do algoritmo proposto. Neste modelo a força de amortecimento é proporcional à velocidade. Um exemplo clássico de aplicação do modelo é o sistema massa-mola-amortecedor com um grau de liberdade descrito pela equação de segunda ordem [17]:

$$
\ddot{x}+2 \zeta \omega_{0} \dot{x}+\omega_{0}^{2} x=0
$$

em que:

$$
\omega_{0}=\sqrt{\frac{\kappa}{m}}
$$

sendo $x$ a posição em função do tempo $t, \zeta$ a taxa de amortecimento ou apenas amortecimento, $\omega_{0}$ a frequência natural de vibração, $m$ a massa e $\kappa$ a constante elástica da mola. 
Assumindo a solução $\mathrm{x}(\mathrm{t})=\mathrm{e}^{\mathrm{jyt}}$ para e equação $(\mathrm{A})(\mathrm{j}=$ número complexo unitário), chega-se a $\gamma$ como dado por:

$$
\gamma=\omega_{0}\left(-\zeta \pm \sqrt{\zeta^{2}-1}\right)
$$

O comportamento do sistema massa-mola-amortecedor depende da solução de $\gamma$, que pode ter duas soluções reais $(\zeta>1)$, uma solução real $(\zeta=1)$ ou duas soluções complexas $(0 \leq \zeta<1)$. Os casos sobreamortecido $(\zeta>1)$ e criticamente amortecido ( $\zeta=1$ ) são não-oscilatórios e irrelevantes para este trabalho. Quando $0 \leq \zeta<1$ o sistema é sub-amortecido e a solução da equação (F) pode ser escrita como:

$$
x(t)=A_{0} \cdot e^{-\xi \omega_{0} t} \cdot \cos (\omega t+\varphi)
$$

em que $\mathrm{A}_{0}$ é a amplitude inicial de vibração, $\varphi$ é a fase inicial da vibração e $\omega$ é a frequência natural amortecida descrita por:

$$
\omega=\omega_{0} \sqrt{1-\xi^{2}}
$$

Esse modelo também é conhecido como sistema linear amortecido com um grau de liberdade [8, 17]. Generalizando para um sistema com $\mathrm{K}$ graus de liberdade, a amplitude de vibração de um determinado ponto da amostra, quando captada por um sensor, é dada por:

$$
x(t)=\left[\sum_{i=1}^{K} A_{i} \cdot e^{-\xi_{i} \omega_{01} t} \cdot \cos \left(\omega_{i} t+\varphi_{1}\right)\right]+R_{w n}
$$

em que $A_{i}, \zeta_{i}, \omega_{0 i}, \omega_{i}, \varphi_{\iota}$ são, respectivamente, amplitude inicial, amortecimento, frequência natural de vibração, frequência natural amortecida e fase inicial do i-ésimo grau de liberdade ou modo de vibração naquele ponto. O termo $\mathrm{R}_{\mathrm{wn}}$ é um ruído descorrelacionado do sinal captado ou gerado pelo sensor, assumido como sendo ruído branco.

O modelo viscoelástico equivalente consiste em empregar as equações do modelo viscoelástico para sistemas que apresentam decremento exponencial da amplitude de vibração, que é um comportamento típico de sistemas viscoelásticos. Contudo estes sistemas também podem apresentar mecanismos de amortecimento não-viscosos, por exemplo, mecanismos anelásticos dependentes da frequência ou da amplitude de vibração. Esta simplificação por um lado desconsidera a possibilidade de ocorrência de mecanismos não-viscosos, mas por outro viabiliza caracterizações experimentais comparativas para corpos de prova com faixa de frequência de ressonância e dimensões similares [18].

\section{Algoritmo desenvolvido}

Conforme ilustrado no fluxograma da Fig. 1, o algoritmo desenvolvido foi estruturado em 05 passos principais visando aproveitar a padronização das condições de contorno mecânicas do ensaio para simplificar o processamento de sinal. Este aproveitamento se deu com a determinação do decremento logarítmico baseando-se no acompanhamento da amplitude das freqüências encontradas no primeiro segmento do sinal, em vez de se aplicar filtros digitais integradores ao redor destas freqüências ou de utilizar transformadas especiais como é usualmente realizado em análise modal $[11,12]$.

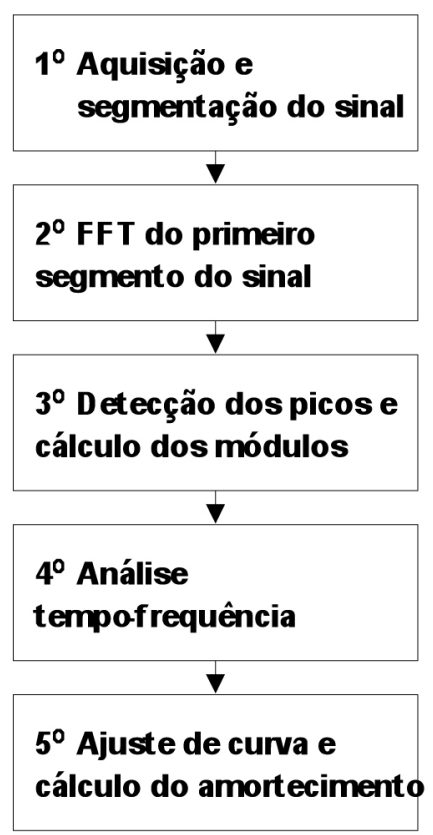

Figura 1: Fluxograma resumido do algoritmo desenvolvido. [Figure 1: Developed algorithm flowchart.]

O primeiro passo consiste na aquisição do sinal e na sua segmentação. A digitalização é realizada através de uma placa de áudio ou pelo hardware onde o algoritmo estiver embarcado. A taxa de amostragem da digitalização deve ser pelo menos o dobro da frequência mais alta que se deseja analisar. A segmentação do sinal é realizada de acordo com a equação:

$$
\mathrm{s}_{\mathrm{h}}(\mathrm{k})=\mathrm{s}(\mathrm{k}+\mathrm{hH}), 0 \leq \mathrm{k}<\left(\mathrm{n}_{\mathrm{s}}-1\right)
$$

em que $h$ é o índice do segmento, $H$ é espaçamento (offset) entre o início de um novo segmento e o início do segmento anterior, e $n$ é o comprimento de cada segmento em número de pontos. A razão entre $H$ e $n_{s}$ determina a sobreposição ou espaçamento entre os segmentos.

O segundo passo consiste na realização da transformada rápida de Fourier (FFT) [19] do primeiro segmento do sinal $\left(s_{1}\right)$ com $n_{s}$ amostras. É no primeiro segmento que as componentes de sinal apresentam a maior amplitude, visto que a resposta acústica do corpo de prova é um sinal transitório com decremento exponencial. Usando a FFT e zero-padding [19], é encontrado o espectro de magnitudes $\left|\mathrm{S}_{1}\left(\mathrm{e}^{\mathrm{jw}}\right)\right|$ do segmento de sinal $\mathrm{s}_{1}(\mathrm{k})$.

O terceiro passo consiste na detecção dos picos do espectro de magnitudes $\left|S_{1}\left(e^{j w}\right)\right|$ e no cálculo dos módulos elásticos. Para isso é calculado o valor médio da derivada do segmento, $\bar{\delta}_{\mathrm{s}_{1}}$, através da equação: 


$$
\bar{\delta}_{\mathrm{s}_{1}}=\frac{2}{\mathrm{n}_{\mathrm{S}}} \sum_{\mathrm{n}=1}^{\frac{\mathrm{n}_{\mathrm{s}}}{2}-1}\left|\mathrm{~S}_{1}(\mathrm{n})-\mathrm{S}_{1}(\mathrm{n}-1)\right|
$$

com $n$ sendo a indexação dos valores de frequência do espectro $\left|S_{1}\left(e^{j w}\right)\right|$. São consideradas possíveis frequências de ressonância todos os picos que possuírem em sua borda de subida inclinação maior que a derivada média.

O quarto passo consiste na análise tempo-frequência do sinal com a montagem de uma matriz que contém a informação de como a amplitude de cada frequência detectada varia com o tempo desde o momento da excitação do corpo de prova. Para o cálculo da FFT dos segmentos $s_{h}$, todos são multiplicados por uma janela flat-top [20] e submetidos ao zero-padding.

O quinto e último passo consiste no ajuste de uma curva sobre a evolução da amplitude de cada frequência $\omega_{i}$ registrada na matriz tempo-freqüência, e no cálculo do respectivo amortecimento a partir do decremento logarítmico da região onde ocorre o decremento exponencial do sinal. Para a realização do ajuste de curva é empregada a equação (N) com o método não-linear por mínimos quadrados proposto por Levemberg-Marquardt [21, 22]. Este método permite selecionar a região a ser priorizada pelo ajuste em função da amplitude através do parâmetro $\sigma$ que pode assumir valores entre 0,1 e 1 .

$$
\mathrm{a}_{\mathrm{i}}(\mathrm{t})=\left(\frac{\mathrm{A}_{0 \mathrm{i}}^{2}}{2} \mathrm{e}^{2 \delta_{\mathrm{i}} \mathrm{t}}+\mathrm{A}_{\mathrm{wn}}^{2}\right)^{\mathrm{\sigma} / 2}
$$

$\mathrm{Na}$ equação $(\mathrm{N})$ empregada pelo método LevembergMarquardt [21, 22] $\mathrm{a}_{\mathrm{i}}$ (t) é a amplitude RMS (Root Mean Square) da frequência $\omega_{\mathrm{i}}$ em função do tempto $t, \delta_{\mathrm{i}}$ é o decremento logarítmico correspondente e $A_{w n}$ o piso de ruído. O decremento logarítmico $\delta_{\mathrm{i}}$ pode ser visualizado como o coeficiente angular da queda de amplitude em escala logarítmica em função do tempo. Determinado $\delta_{i}, o$ amortecimento $\zeta_{\mathrm{i}}$ é calculado através da relação:

$$
\zeta_{\mathrm{i}}=\delta_{\mathrm{i}} / \omega_{\mathrm{i}}
$$

A execução destes cinco passos é muito rápida e tem a duração determinada essencialmente pela duração da resposta acústica emitida pelo corpo de prova.

\section{Aparato experimental}

Para a aquisição de sinal foi empregado neste trabalho um sensor acústico capacitivo com largura de banda de 20 $\mathrm{Hz}$ a $20 \mathrm{kHz}$. Para a digitalização do sinal foi empregada uma placa de aquisição e processamento de sinais com taxa de aquisição de até $96 \mathrm{ksp} / \mathrm{s}$ (ksamples/second - quilo amostras/segundo) e quantização em 24 bits.

As caracterizações foram realizadas utilizando o suporte de corpo de prova de precisão para barras retangulares modelo Sonelastic ${ }^{\circledR}$ SP-B (ATCP Engenharia Física) que impõem as condições de contorno ótimas para o modo de vibração flexional fundamental. Neste suporte a amostra é apoiada exatamente sobre os nós de vibração flexional

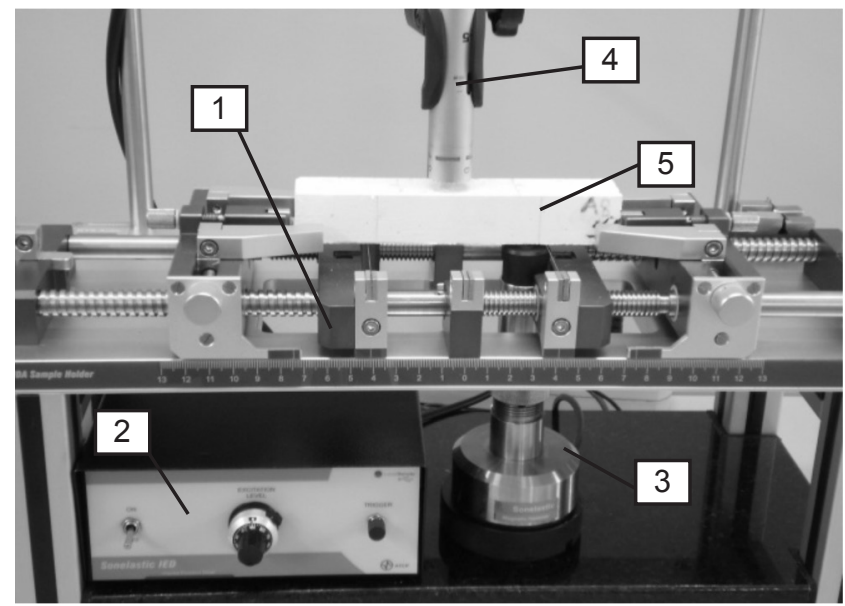

Figura 2: Sistema para suporte, excitação e captura de sinal. 1: dispositivo de posicionamento automático; 2 e 3: atuador Sonelastic ${ }^{\circledR}$ IED; 4: captador acústico; 5: corpo de prova.

[Figure 2: Apparatus for sample holder, excitation and signal acquisition. 1: automatic positioning device; 2 and 3: Sonelastic IED actuator; 4: acoustic sensor; 5: specimen.]

fundamental por fios metálicos na posição 0,224L, em que L é o comprimento da barra. O ajuste da posição é realizado automaticamente por um sistema mecânico fundamentado no comprimento total do corpo de prova (Fig. 2). É importante que o sistema de excitação e captação do sinal exerça a mínima influência possível sobre a vibração flexional fundamental. A excitação mecânica é provida pelo atuador eletromagnético Sonelastic ${ }^{\circledR}$ IED.

\section{EXEMPLO DE APLICAÇÃO}

O algoritmo desenvolvido programado em MATLAB $\mathbb{R}$ foi empregado em conjunto com o aparato experimental para a determinação simultânea do amortecimento, do módulo de

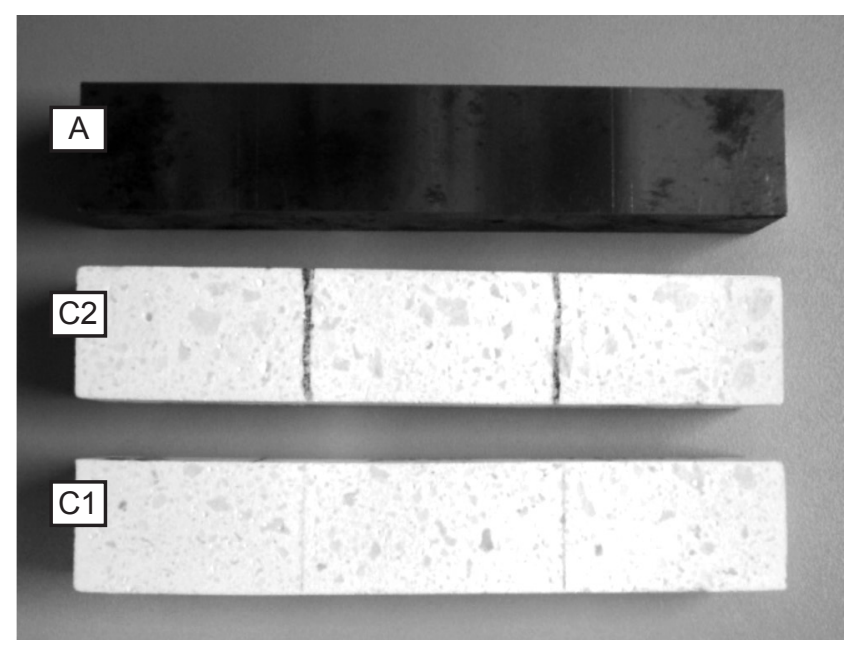

Figura 3: Corpos de prova: Aço SAE 1020 (A), concreto refratário sem choque térmico (C1) e concreto refratário com choque térmico (C2).

[Figure 3: Specimens: Steel SAE 1020 (A), refractory castable without thermal shock (C1) and refractory castable with thermal shock (C2).] 
Young, do módulo de cisalhamento e da razão de Poisson de um concreto refratário de alta alumina e de um aço SAE 1020. O concreto refratário foi caracterizado com e sem dano por choque térmico. Os corpos de prova caracterizados foram preparados no formato de barras retangulares e estão mostrados na Fig. 3. Este formato é padrão para ensaios mecânicos de resistência mecânica, portanto muito comum.

$\mathrm{Na}$ Tabela I são apresentadas as dimensões e a massa dos corpos de prova com as respectivas incertezas. Os corpos de concreto foram conformados por vibração em moldes metálicos e tiveram a face mais grosseira retificada (a mesma face que está sendo mostrada pela foto da Fig. 3; a amostra de aço teve as quatro faces maiores retificadas.

Tabela I - Dimensões e massa dos corpos de prova. [Table I - Specimens dimensions and mass.]

\begin{tabular}{lccc}
\hline \multirow{2}{*}{ Parâmetro } & \multicolumn{3}{c}{ Corpo de prova } \\
& A & C1 & C2 \\
\hline $\begin{array}{l}\text { Comprimento } \\
(\mathrm{mm})\end{array}$ & $150,0 \pm 0,10$ & $149,0 \pm 0,25$ & $148,9 \pm 0,25$ \\
Largura (mm) & $25,02 \pm 0,03$ & $25,18 \pm 0,25$ & $25,57 \pm 0,25$ \\
Altura (mm) & $25,01 \pm 0,03$ & $23,83 \pm 0,30$ & $23,68 \pm 0,30$ \\
Massa (g) & $733,9 \pm 0,10$ & $284,2 \pm 0,10$ & $288,7 \pm 0,10$ \\
\hline
\end{tabular}

\section{Aquisição e segmentação do sinal}

Conforme o fluxograma da Fig. 1 , o $1^{\circ}$ passo do algoritmo é a aquisição e a segmentação do sinal, que foram realizadas de acordo com os parâmetros da Tabela II.

Os parâmetros de aquisição e segmentação foram ajustados para que o número total de segmentos se mantivesse em torno de 250 para os três corpos de prova. O tempo de aquisição foi ajustado para que o sinal fosse captado até pelo menos no momento em que se torna menor que o piso de ruído. Desta forma, tem-se toda a informação possível para o posterior ajuste de curva sem se sobrecarregar o processamento computacional. Nota-se que a duração do sinal é muito maior para o aço, devido ao seu baixo amortecimento comparativamente ao do concreto.

Na Fig. 4 é mostrado um exemplo de sinal adquirido,

Tabela II - Parâmetros da aquisição e segmentação dos sinais.

[Table II - Signals acquisition and segmentation parameters.]

\begin{tabular}{lcc}
\hline \multirow{2}{*}{ Parâmetros } & \multicolumn{2}{c}{ Corpo de prova } \\
& $\mathrm{A}$ & $\mathrm{C} 1 \mathrm{e} \mathrm{C} 2$ \\
\hline Taxa de amostragem (ksp/s) & 48 & 96 \\
Tempo de aquisição (s) & 5,46 & 0,683 \\
Offset (pontos) & 1.024 & 256 \\
Comprimento do segmento (pontos) & 8.192 & 2.048 \\
Número de segmentos & 249 & 249 \\
\hline
\end{tabular}

mais especificamente o sinal da amostra de concreto sem dano por choque térmico $(\mathrm{C} 1)$.

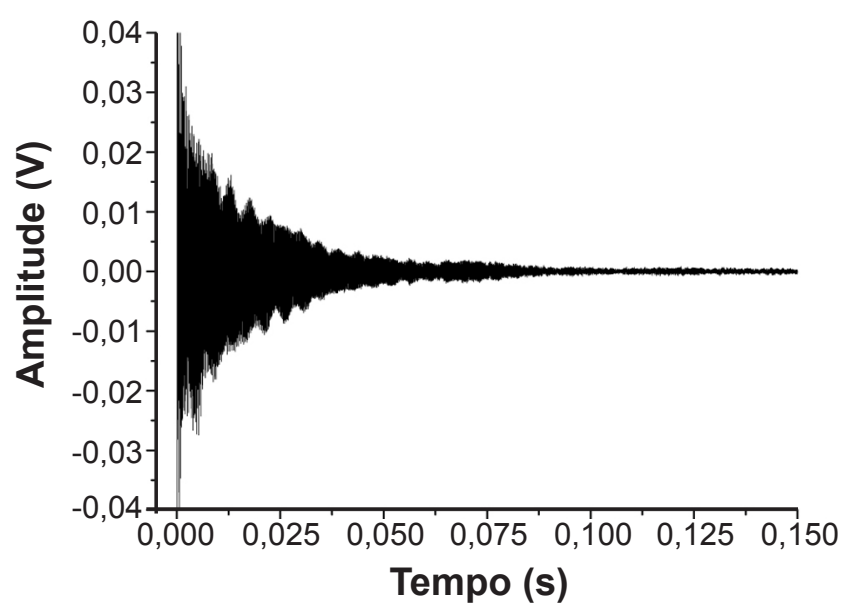

Figura 4: Exemplo de sinal adquirido correspondente a resposta acústica do corpo de prova à excitação provida pelo pulsador. Este sinal especificamente é do concreto sem dano (C1).

[Figure 4: Example of signal acquisition which corresponds to the acoustic response induced by the actuator action over the specimen. This signal is correspondent to the castable without thermal shock (C1) acoustical response.]

\section{Detecção das freqüências e cálculo dos módulos}

O segundo e o terceiro passo consistem na obtenção do espectro de freqüências do sinal contido no primeiro segmento, na detecção automática das freqüências presentes e no cálculo dos módulos elásticos. Na Fig. 5 é mostrado um exemplo de espectro, mais especificamente da amostra de concreto sem dano por choque térmico $(\mathrm{C} 1)$, com as frequências detectadas na faixa de interesse destacadas com

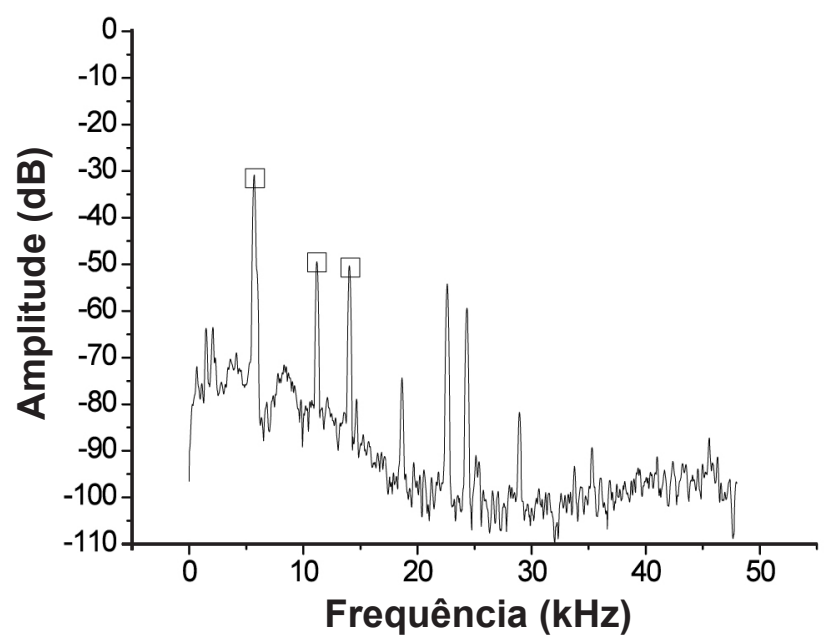

Figura 5: Exemplo de espectro de freqüências correspondente a FFT do primeiro segmento do sinal. Este espectro em particular é do concreto sem dano $(\mathrm{C} 1)$.

[Figure 4: Example of frequency spectrum correspondent to the first signal segment FFT. This spectrum is correspondent to the castable without thermal shock (C1) acoustical response.] 
quadrados. A amplitude de referência para as escalas em $\mathrm{dB}$ é amplitude máxima aceita pela placa de aquisição $(2 \mathrm{~V}$ pico-a-pico).

Na Tabela III são apresentadas as freqüências detectadas na faixa de interesse (até $15 \mathrm{kHz}$ ); acima de $15 \mathrm{kHz}$ se encontram $\mathrm{s}$ freqüências harmônicas destes corpos e estas não são necessárias para os cálculos dos módulos elásticos.

Tabela III - Principais freqüências detectadas em cada corpo de prova.

[Table III - Main detected frequencies for each specimen.]

\begin{tabular}{cccc}
\hline \multirow{2}{*}{ Pico } & \multicolumn{3}{c}{ Amostras } \\
& $\mathrm{A}$ & $\mathrm{C} 1$ & $\mathrm{C} 2$ \\
\hline $1^{\circ}(\mathrm{Hz})$ & $5.381,7$ & $5.684,6$ & $3.580,7$ \\
$2^{\circ}(\mathrm{Hz})$ & $9.910,5$ & $11.187,6$ & $8.324,5$ \\
$3^{\circ}(\mathrm{Hz})$ & $13.104,8$ & $14.035,4$ & $9.168,5$ \\
\hline
\end{tabular}

Com o conhecimento da massa, das dimensões dos corpos de prova (Tabela I) e das suas frequências de ressonância (Tabela III são calculados os módulos elásticos a partir das equações de Pickett [14], que também permitem a identificação do modo de vibração a que pertence cada frequência a partir da razão entre elas. Estas razões dependem essencialmente das proporções geométricas do corpo de prova. No caso de barras retangulares de secção quadrada com aresta 6 vezes menor que o comprimento, a primeira frequência $\left(1^{\circ}\right.$ pico) é o modo flexional fundamental e a segunda ( $2^{\circ}$ pico) o modo torcional fundamental. Na Tabela IV são apresentados os resultados obtidos para os módulos elásticos. As incertezas apresentadas nos resultados da Tabela IV foram calculadas a partir da propagação da incerteza das dimensões, da massa e das equações de Pickett.

Tabela IV - Módulos elásticos obtidos.

[Table IV - Obtained elastic moduli.]

\begin{tabular}{cccc}
\hline $\begin{array}{c}\text { Módulo } \\
\text { elástico }\end{array}$ & $\mathrm{A}$ & $\mathrm{C} 1$ & $\mathrm{C} 2$ \\
\hline $\begin{array}{c}\text { Young }(\mathrm{GPa}) \\
\text { Cisalhamento } \\
\text { (GPa) }\end{array}$ & $206 \pm 1$ & $98 \pm 3$ & $39,6 \pm 1,3$ \\
Razão de \\
Poisson & $0,26 \pm 0,01$ & 0,18 & $-0,16 \pm 0,04$ \\
\hline
\end{tabular}

O quarto passo é a análise tempo-frequência do sinal, que consiste na obtenção do espectro de freqüências via FFT para cada um dos segmentos do sinal, de modo que seja possível o acompanhamento do decremento logarítmico de cada frequência presente, e a partir desta informação, realizar o cálculo dos respectivos amortecimentos. Na Fig. 6 é apresentado como exemplo o espectrograma da resposta acústica do corpo de prova de aço. O perfil do decremento é visto como uma reta e não como uma exponencial porque a escala de amplitude é logarítmica; o parâmetro taxa de decremento logarítmico $\left(\delta_{\mathrm{i}}\right)$ pode ser visualizado como o coeficiente angular da rampa formada pelo decaimento do sinal.

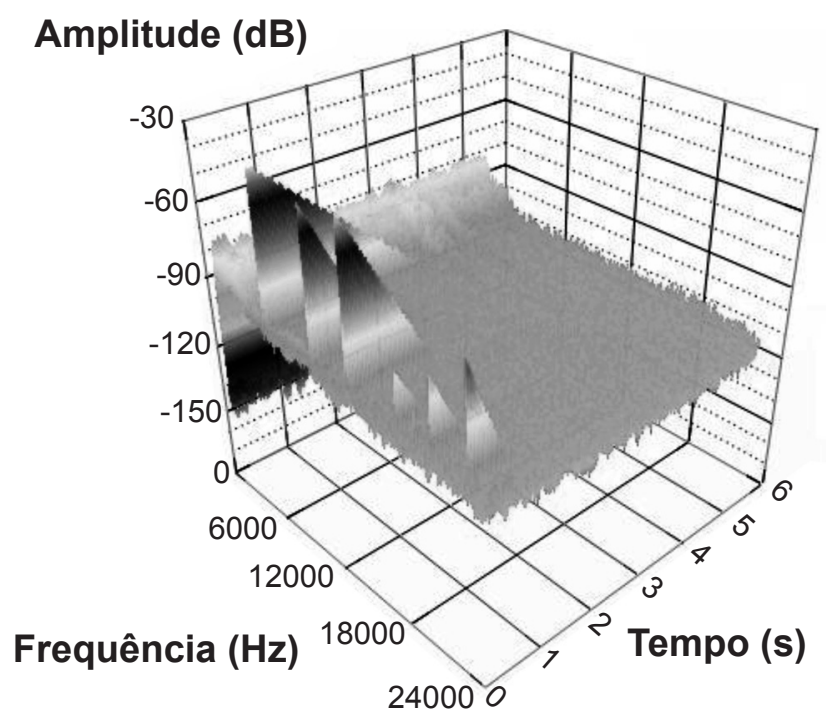

Figura 6: Espectrograma da resposta acústica do corpo de prova de aço SAE 1020 (A).

[Figure 6: Acoustic spectrogram of the steel specimen (A).]

O quinto passo consiste no ajuste de curva sobre o decremento da amplitude da frequência de interesse em função do tempo, empregando o algoritmo não-linear por mínimos quadrados Levemberg-Marquardt [16, 17]. Esta frequência corresponde normalmente ao modo de vibração mais favorecido pelas condições de contorno do suporte de corpo de prova, no caso do aparato experimental empregado

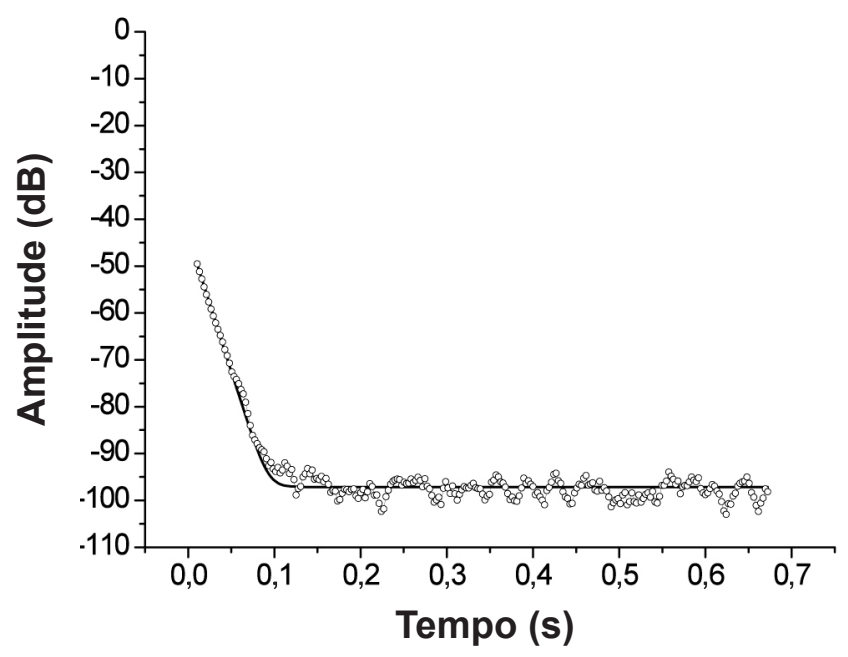

Figura 7: Ajuste de curva sobre o decremento logarítmico do $1^{\circ}$ pico (modo flexional fundamental) do concreto sem dano por choque térmico (C1).

[Figure 7: Curve fitting of the logarithm decay of the $1^{\circ}$ peak (fundamental flexional mode) of the castable without thermal shock (C1).] 
para este trabalho, o modo flexional fundamental. Na Fig. 7 é apresentado um exemplo de ajuste de curva; o coeficiente angular da região de queda linear do ajuste é o parâmetro taxa de decremento logarítmico.

Na Tabela V são apresentados os resultados encontrados para o amortecimento do modo flexional fundamental dos concretos e do aço empregando o algoritmo desenvolvido. Para efeito de comparação, é apresentado também o resultado realizando-se o ajuste sobre a amplitude do sinal no domínio do tempo.

Tabela V - Amortecimento obtido pelo algoritmo desenvolvido (Algo.D.) e pelo ajuste no domínio do tempo (Ref.).

[Table V-Damping obtained by the proposed algorithm and by the fitting on time domain.]

\begin{tabular}{cccc}
\hline \multirow{2}{*}{$\left(\mathrm{x} 10^{-6}\right)$} & $\mathrm{A}$ & $\mathrm{C} 1$ & $\mathrm{C} 2$ \\
\hline Algo.D. & 72,8 & 1.249 & 4.366 \\
Ref. & 104,4 & 1.357 & 6.820 \\
\hline
\end{tabular}

Nota-se que o amortecimento obtido com o algoritmo desenvolvido é menor que o obtido do decremento do sinal no domínio do tempo; o que era esperado, visto que este elimina a influência indesejável dos modos de vibração menos favorecidos pelas condições de contorno mecânicas, mas que estão inevitavelmente presentes e são necessários para o cálculo simultâneo do módulo de cisalhamento e da razão de Poisson.

\section{FAIXA DE MEDIÇÃO E VALIDAÇÃO}

\section{Faixa de medição}

Os extremos para a medição do amortecimento pelo método do decremento logarítmico são 0 e 1 . O extremo igual a 0 , que é o menor valor possível de amortecimento, não ocorre dissipação de energia e o sistema vibra indefinidamente com amplitude constante. No extremo igual a 1 o sistema é criticamente amortecido e retorna ao equilíbrio após a excitação sem completar um ciclo de oscilação; a medição do amortecimento com o algoritmo neste extremo, ou muito próximo a ele, não é possível porque a frequência do sinal não é detectável. Portanto, determinar a faixa de medição consiste essencialmente em estimar o quão próximo é possível chegar do limite onde o amortecimento é igual a 1, em função da frequência e amplitude do sinal, e dos parâmetros da aquisição e do processamento. Como o algoritmo é fundamentado no ajuste de uma curva sobre a evolução da amplitude da frequência de interesse $\omega_{\mathrm{i}}$ ao longo dos segmentos $\mathrm{S}_{\mathrm{h}}$, o amortecimento máximo mensurável $\zeta_{i \max }$ para uma determinada frequência $\omega_{i}$ é limitado pela razão entre a duração do sinal e a duração do primeiro segmento mais o espaçamento entre este e o(s) segmento(s) subsequente(s). Esta razão deve ser maior ou igual a 1. Se esta razão for inferior a 1 , o que significa que duração do sinal não foi suficiente para a obtenção de pelo menos dois pontos para o preenchimento da matriz tempo-frequência, não é possível o ajuste da curva para a determinação do decremento logarítmico, e consequentemente, o cálculo do amortecimento. Adi-cionalmente, os segmentos $S_{h}$ não podem ser reduzidos indefinidamente porque a redução degrada a precisão na determinação da frequência e da amplitude, visto que a precisão em frequência é diretamente proporcional à taxa de amostragem e inversamente proporcional ao número de pontos do segmento.

Da mesma forma que o cálculo do amortecimento, a faixa mensurável de amortecimento depende da frequência $\omega$ (equação(O)). Portantoéconveniente basearo equacionamento de $\zeta_{i \max }$ em termos do decremento logarítmico máximo $\delta_{\mathrm{i} \text { max }}$ :

$$
\zeta_{i \max }=\frac{\delta_{i \max }}{\omega_{i}} \frac{1}{20 \cdot \log (\mathrm{e})}
$$

e o decremento logarítmico máximo $\delta_{i \max }$ em termos da amplitude do sinal relativa ao piso de ruído e da duração do primeiro segmento mais o(s) intervalo(s) de tempo entre o primeiro e os $N$ - 1 segmento(s) conseguinte(s):

$$
\delta_{i \max }=\frac{\left|\mathrm{A}_{0}-\mathrm{A}_{\mathrm{wm}}\right|}{\left[\frac{\mathrm{n}_{\mathrm{S}}+(\mathrm{N}-1) \mathrm{H}}{\mathrm{f}_{\mathrm{a}}}\right]}
$$

nas equações (Q) e (P) $A_{0}$ é a amplitude inicial do sinal (em $\mathrm{dB}), A_{w n}$ o piso de ruído (em dB), $f_{a}$ a taxa de aquisição ou frequência de amostragem do sinal e $N$ o número mínimo de pontos que se deseja obter para realizar o ajuste da curva ( $N$ deve ser $\geq 2$ ). A inserção da constante $1 / 20 . \log (\mathrm{e})$ na equação $(\mathrm{Q})$, em que "e" é o número de Neper, deve-se à compatibilização da amplitude (em dB na base 10) com a base "e" do método do decremento logarítmico.

Com o amortecimento máximo mensurável equacionado, o próximo passo para estimar a faixa de medição é realizar o cálculo para as condições experimentais típicas. $\mathrm{Na}$ Tabela VI são apresentados valores típicos para os parâmetros determinantes de $\delta_{i \max }$ nas situações em que o algoritmo desenvolvido será inicialmente aplicado e para

Tabela VI - Valores típicos dos parâmetros que determinam $\delta_{i \max }$.

[Table VI - Typical values of the parameters which determine $\delta_{\text {imax }}$.

\begin{tabular}{cc}
\hline Parâmetro & Valor típico \\
\hline $\mathrm{n}_{\mathrm{s}}$ & 1.024 pontos \\
$\mathrm{H}$ & 256 pontos \\
$\mathrm{f}_{\mathrm{a}}$ & $96 \mathrm{ksp} / \mathrm{s}$ \\
$\mathrm{N}$ & 3 \\
$\left|\mathrm{~A}_{0}-\mathrm{A}_{\mathrm{ps}}\right|$ & $36 \mathrm{~dB}$ \\
\hline$\delta_{\mathrm{i} \max }$ & $2.250 \mathrm{~dB} / \mathrm{s}$ \\
\hline
\end{tabular}


que possa ser facilmente embarcado em uma eletrônica com processamento de sinal voltado para áudio.

Conhecendo $\delta_{i \max }$, pode-se calcular o amortecimento máximo em função da frequência empregando-se a equação (Q); quanto maior a frequência, menor o amortecimento máximo mensurável. Para a frequência de $5 \mathrm{kHz}, \operatorname{com} \delta_{i \max }=2.250 \mathrm{~dB} / \mathrm{s}$, o amortecimento máximo é da ordem de $10^{-2}$, que é um valor alto para materiais cerâmicos em temperatura ambiente. Para $500 \mathrm{~Hz}$ o máximo atinge $10^{-1}$. Para o teste de materiais cerâmicos, por exemplo, concretos refratários e abrasivos, a faixa de frequência usual está entre 2 e $6 \mathrm{kHz}$ e de amortecimento entre $10^{-5} \mathrm{e} 10^{-2}$. Contudo para amostras pequenas $(<10 \mathrm{~cm})$, a frequência pode atingir valores superiores.

Portanto, para frequências de até $5 \mathrm{kHz}$, a faixa de medição do amortecimento pelo algoritmo proposto para materiais cerâmicos é de aproximadamente de 0 a $10^{-2}$, sendo este valor inversamente proporcional à frequência de acordo com a equação (Q) e com a ressalva de que esta faixa pode variar em função dos parâmetros da aquisição e do processamento do sinal.

\section{Validação}

O algoritmo desenvolvido foi testado com a simulação de medições empregando sinais transitórios sintetizados com frequência e amortecimento bem definidos. Para sintetizar os sinais foi utilizado um gerador de forma de onda arbitrária modelo HP-33120A (Hewlett-Packard). Foram escolhidos amortecimentos próximos ao limite de medição, entre aproximadamente $10^{-1}$ e $10^{-3} \mathrm{p} / \mathrm{N}=3$, na faixa de frequência de $200 \mathrm{~Hz}$ a $20 \mathrm{kHz}$. A amplitude do sinal simulado com relação ao piso de ruído foi ajustada para $44 \mathrm{~dB}$. Também foram simuladas medições com amortecimento igual a zero na mesma faixa de frequência. Os resultados mostraram que para sinais com valores de amortecimento de até $10^{-2}$ e frequências entre 500 e $10 \mathrm{kHz}$, o erro médio e o desvio padrão da determinação do amortecimento é inferior a $1 \%$ e para a determinação da frequência inferior a $0,1 \%$. Para condições extremas, tendendo ao limite mensurável teórico, o erro médio e o desvio padrão foram de até $4 \%$ para o amortecimento e $8 \%$ para a frequência. Estes valores de erro estão compatíveis ou inferiores ao descritos na literatura para métodos de medição similares [12].

\section{CONCLUSÕES}

Foi desenvolvido um algoritmo fundamentado no método do decremento logarítmico, no modelo viscoelástico equivalente e na análise tempo-frequência, para a determinação do amortecimento de materiais cerâmicos; e este incorporado à técnica das frequências naturais de vibração via excitação por impulso. $\mathrm{O}$ algoritmo, em associação com o aparato experimental empregado, se mostrou preciso na análise de sinais sintetizados com frequência e amortecimento nas faixas típicas das amostras de materiais cerâmicos (frequência de até $6 \mathrm{kHz}$ e amortecimento de até $10^{-2}$ ), na caracterização de concreto refratários de alta alumina e na caracterização de um aço SAE 1020. Graças à análise tempo- freqüência, o algoritmo foi eficiente na medição isolada do amortecimento de cada frequência presente nas respostas acústica e apresentou erros compatíveis ou inferiores aos descritos na literatura para sistema de análise modal que compartilham os mesmos princípios. É interessante notar que o aumento percentual do amortecimento do concreto caracterizado devido ao choque térmico foi aproximadamente uma ordem de grandeza superior à queda percentual dos módulos elásticos $(\sim+350 \%$ vs. $-40 \%$ ), o que evidencia a sensibilidade deste parâmetro para a avaliação do dano por choque térmico nesta categoria de materiais cerâmicos. O choque térmico induziu a formação de trincas e micro-trincas que enfraqueceram o material e elevaram o atrito interno. A integração do algoritmo não comprometeu a praticidade ou alterou os procedimentos da técnica das frequências naturais de vibração via excitação por impulso. Com esse avanço, a técnica passa a informar um parâmetro importante dos materiais cerâmicos a partir do mesmo procedimento experimental usado até então, sem a necessidade de empregar aparatos experimentais adicionais específicos para análise modal.

\section{AGRADECIMENTOS}

Ao CNPq (Procs. 301073/2006-6, 303061/20090 e 141868/2008-2) e à FINEP (Proj. 4028-6) pelo apoio financeiro, e às empresas Elfusa e Almatis pela doação da matéria prima.

\section{REFERÊNCIAS}

[1] B. J. Lazan, Damping of materials and members in structural mechanics, Pergamon Press, Oxford (1968).

[2] R. Dieterle, H. Banchmann, Int. Assoc. Bridge and Structural Eng. Report Working Com. 34 (1981) 69.

[3] J. A. Coppola, J. Am. Ceram. Soc. 56, 4 (1973) 214.

[4] T. Tonnesen, R. Telle, Ceram. Forum Int. 84, 9 (2007) 132.

[5] D. P. Hasselman, J. Am. Ceram. Soc. 46, 11 (1963) 535.

[6] W. D. Kingery, J. Am. Ceram. Soc. 38, 3 (1955) 3.

[7] S. H. Chowdhury, PhD Thesis, Griffith University, Australia (1999).

[8] C. W. Silva, Vibration damping, control, and design, Taylor \& Francis Group, Vancouver, Canada (2007) 634p.

[9] A. Puskar, Internal friction of materials, Cambridge Int. Sci. Publ., Cambridge, Inglaterra (2001) 342p.

[10] S. Gade, H. Herlufsen, H. Konstantin-Hansen, Application notes BO 0177-11, Brüel\&Kraer (2002).

[11] Damping Measurements, Technical Rev.2, Brüel\&Kraer (1994).

[12] Digital Filter Techniques vs. FFT Techniques for Damping Measurements, Tech. Rev. 1, Brüel\&Kraer (1994). [13] Standard Test Method for Dynamic Young's Modulus, Shear Modulus, and Poisson's Ratio by Impulse Excitation of Vibration, ASTM E 1876-07, ASTM Int. (2007). 
[14] G. Pickett, Proc. Am. Soc. Test. Mater. 45 (1945) 846.

[15] R. Morrel, Measuring elastic properties of advanced technical ceramics - A review, UK Nat. Phys. Lab. Report 42 (1996).

[16] Operating Instructions for the GrindoSonic MK5 "Industrial" Instrument, J. W. Lemmens, Inc.

[17] D. Thorby, Structural Dynamics and Vibrations in Practice - An Engineering Handbook, Elsevier Ltd., Oxford, Inglaterra (2008) 420p.

[18] A. H. A. Pereira, G. M. Fortes, B. Schickle, T. Tonnesen, B. Musolino, C. D. B. Maciel, J. A. Rodrigues, Cerâmica 56 (2010) 311 .
[19] A.V. Oppenheim, R. W. Schafer, J. R. Buck, DiscreteTime Signal Processing, $2^{\text {nd }}$ Ed., Prentice-Hall, Inc., Upper Saddle River, EUA (1998) 870p.

[20] A. F. Harvey, M. Cerna, Application Note 041, Nat. Instr. Corp., EUA (1993).

[21] K. Madsen, H. B. Nielsen, O. Tingleff, Methods for Non-Linear Least Squares Problems, $2^{\text {nd }}$ Ed. Informatics and Mathematical Modeling, Dinamarca (2004).

[22] L. Finschi, Technical Report, Institute for Operations Research, ETH Zürich (1996), http://www.ifor.math.ethz. $\mathrm{ch} /$ staff/finschi/LevMar/LevMar.pdf

(Rec. 28/06/2011, Ac. 22/08/2011) 